\title{
At-Wavelength Interferometry of High-NA Diffraction-Limited EUV Optics
}

\author{
Kenneth A. Goldberg*, Patrick Naulleau*, Senajith Rekawa*, Paul Denham*, \\ J. Alexander Liddle*, Erik Anderson*, Keith Jackson*, Jeffrey Bokor ${ }^{* \dagger}$, David Attwood ${ }^{* \dagger}$ \\ ${ }^{*}$ Center for X-Ray Optics, Lawrence Berkeley National Laboratory, Berkeley, CA 94720 \\ $\dagger$ EECS Department, University of California, Berkeley, CA 94720
}

\begin{abstract}
Recent advances in all-reflective diffraction-limited optical systems designed for extreme ultraviolet (EUV) lithography have pushed numerical aperture (NA) values from 0.1 to 0.3 , providing Rayleigh resolutions of 27-nm. Worldwide, several high-NA EUV optics are being deployed to serve in the development of advanced lithographic techniques required for EUV lithography, including the creation and testing of new, high-resolution photoresists. One such system is installed on an undulator beamline at Lawrence Berkeley National Laboratory's Advanced Light Source. Sub$\AA$-accuracy optical testing and alignment techniques, developed for use with the previous generations of EUV lithographic optical systems, are being extended for use at high NA. Considerations for interferometer design and use are discussed.
\end{abstract}

\section{INTRODUCTION}

Earlier generations of all-reflective, Mo/Si-multilayer-coated EUV imaging optics have included two-mirror Schwarzschild designs [1], and large-scale, four-mirror, ring-field systems [2]. The most recently fabricated EUV lithographic optical systems, called the Micro Exposure Tool (MET), are two-mirror, 0.3 NA, axially-symmetric systems with an annular pupil and a modest, $1 \times 3 \mathrm{~mm}$ field of view: they operate with an object plane tilted at $4^{\circ}$ to accommodate a reflective reticle.

For lithographic pattern transfer, figure aberrations on the scale of $0.1 \mathrm{~nm}$ affect imaging performance. Although it is at or beyond the limits of today's interferometers, RMS accuracy surpassing 0.1-nm has become a cornerstone requirement for continued progress in this field [3]. Techniques in high accuracy interferometry for short-wavelength optical system alignment and characterization are being developed on undulator beamline 12.0 at the Advanced Light Source at Lawrence Berkeley National Laboratory [4]. To date, the primary wavelengths of interest have been in the 13-14 $\mathrm{nm}(88-95 \mathrm{eV})$ range, yet these techniques are applicable to a range of wavelengths where short coherence lengths or the unavailability of reference surfaces preclude the use of more conventional interferometer designs. In this paper we present an overview of interferometer designs, and recent activities.

\section{EUV INTERFEROMETERS}

In our measurements of EUV optics, two interferometer configurations have proven to be most successful: the phase-shifting point-diffraction interferometer (PS/PDI) [5] and the cross-grating lateral-shearing interferometer (LSI) [6]; each design has specific advantages and disadvantages. In addition to these interferometers, a prototype EUV Hartmann wavefront sensor has been operated successfully at low NA values [7]. As implemented in our laboratory, these interferometers are designed to measure wavefront aberrations in two dimensions and require relatively high coherent flux. Similar designs with relaxed coherence and brightness requirements have been proposed for measuring aberrations in one dimension at a time [8].

Unlike many conventional interferometer designs, these interferometers include no re-imaging optics: light propagates to a detector placed in the far-field. The lensless configuration circumvents the potential for errors caused by aberrations in re-imaging optics. However, geometric path length differences between the test and reference beams can be significantly larger than the aberrations of interest. Therefore careful null testing is required to account for systematic, compensable measurement errors [9]. Furthermore, coordinate distortion from the projection of spherical waves onto a planar detector must be accounted for in the data analysis. 


\section{The Phase-Shifting Point-Diffraction Interferometer}

The PS/PDI [1,10-12] has become the de facto accuracy standard for optical system measurements conducted in our laboratory. Pinhole diffraction is used to create spherical reference waves of exceptionally high quality. As shown schematically in Fig. 1a, an objectplane pinhole illuminates the entrance pupil; in the image plane, a second pinhole in an opaque membrane with open-stencil features produces a spherical reference wave that interferes with the aberrated test beam. A nearby window in the image-plane mask transmits the lowspatial-frequency components of the test beam without attenuation.

There are a number of important considerations for the design and use of the PS/PDI. The tradeoff between the pinhole size (and hence its transmission) and the quality of the test beam is a persistent concern. The desire for high-quality reference waves leads to the selection of smaller pinhole sizes where the relative transmission can be low. In order to match the intensities of the test and reference beams, and maintain high fringe contrast for a good signal-to-noise ratio in the measurement, the grating beam-splitter's duty cycle (fraction of open area) can be adjusted. Using an $80 \%$ open area in the grating, for example, produces a relative power difference between the zeroth and first diffracted orders of approximately $31: 1$, compared to approximately 4:1 for the 50\%-open grating [13]. Using the strong zeroth-order as the pinhole-diffracted reference beam brings the two beams closer to parity [14]. The amount of light transmitted through the pinhole also has a strong dependence on the quality of the optical system under test. As alignment or optical quality improves, the Strehl ratio approaches unity. Because the peak intensity at focus dictates the amount of light available for the reference beam's pinhole transmission, as the quality or alignment of the optic under test increases, the measurement becomes easier to perform.

As a rule of thumb, the required pinhole sizes are comparable to or smaller than the resolution of the optical systems under test. For the MET, this requirement translates to $25-40-\mathrm{nm}$ diameter pinholes. To meet the mask opacity requirements, we selected 130-nm-thick $\mathrm{Ni}$ as the absorber material: it has one of the highest available extinction coefficients in this wavelength range (the attenuation length is $14.8 \mathrm{~nm}$ [15]). Pinholes of this high aspect ratio (as high as 5:1) have been fabricated using electron beam lithography on LBNL's Nanowriter. [16] In this size and aspect-ratio domain, scalar diffraction theory fails to adequately predict the transmission efficiency or wavefront quality from these pinholes. We have begun a project comparing angle-resolved diffraction measurements, conducted on arrays of nominally identical pinholes, with modeling performed using TEMPEST 3D, a vector electro-magnetic field simulation program [17].

Another design consideration for the PS/PDI is the desired spatial frequency bandwidth. A wider test-beam window transmits a greater range of spatial frequencies, but necessitates a greater image-plane separation between the test and reference beams, and hence a higher number of fringes across the pupil. In our measurements, we typically choose to have between 70 and 80 fringes across the pupil. For small NA values, the number of fringes is given approximately by $N_{\text {fringes }} \approx 2 s N A / \lambda$ [14], where $s$ is the image-plane beam separation and $\lambda$ is the wavelength. An additional restriction is placed on the window width to minimize measurement noise from the overlap of the test and reference beams: the window width must be less than two-thirds of the beam separation distance (in the direction of the beam separation) [9]. However, a number of geometric systematic errors increase in proportion to the beam separation distance [14] so a compromise value must be found. We have used 5- $\mu \mathrm{m}$ separation for $0.1 \mathrm{NA}$, and $1.7-\mu \mathrm{m}$ for $0.3 \mathrm{NA}$, scaling to keep the number of fringes roughly constant.

The most challenging aspect of using the PS/PDI is the alignment of the reference beam onto the image-plane pinhole. Both the lateral and longitudinal alignments are critical. The pattern of open-stenciled features in our imageplane masks typically include arrays of window-pinhole features located at each field point where measurements take 
place [18]. Within the arrays, a range of pinhole sizes from undersized to oversized guarantees that optimally-sized pinholes will be present. Unambiguous alignment marks in the mask [19] provide position feedback.

Alignment typically proceeds as follows. First the test beam is aligned to pass through the center of a window. Then the beam is moved to the position of the pinhole and carefully aligned until pinhole-diffracted light is visible. At this point, the transmission grating beamsplitter is introduced. If the alignment has not been disturbed, the zerothorder beam will fall onto the pinhole, while one of the first-order beams will pass through the center of the window. Fine alignment is performed observing the quality of the fringes across the pupil, in addition to a real-time Fouriertransform alignment method, based on off-axis Fourier-transform holography, and the observation of a global fringe contrast parameter [20]. The small sizes of the image-plane pinholes (as small as 25-30-nm in the $0.3 \mathrm{NA}$ tests) makes alignment of image-plane components challenging even for high quality translation stages. Owing to the system demagnification, it may be easier to perform fine alignment by adjusting the object pinhole. Since the zeroth order beam can often be easily identified by its relative brightness, it may be possible to leave the grating beamsplitter in the beam continuously, and eliminate one of the alignment steps. The beam separation distance can be controled by adjusting the longitudinal position of the grating.

Systematic aberrations that follow on the beam separation vector can be isolated from the underlying wavefront aberrations and subtracted. In our designs, each window is accompanied by two pinholes, placed 90 degrees apart with respect to the center of the window. Rotating the grating by 90 degrees likewise changes the fringe orientation and affects the geometric aberrations in a predictable manner.

\section{The Cross-Grating Lateral Shearing Interferometer}

The LSI (see Fig. 1b) has a number of advantages that make it well suited to the alignment of short-wavelength optical systems where aberration magnitudes are modest but can be much larger than in the PS/PDI. Compared with the PS/PDI, LSI alignment is substantially easier. The transmission gratings can be fabricated on large windows (e.g. silicon-nitride) and positioned in the beam in the vicinity of the focal plane. To optimize the fringe contrast and minimize measurement complications, the grating, perpendicular to the central ray, should occupy a Talbot plane, given approximately by $z \approx \pm n d^{2} / \lambda[6]$, where $z$ is the longitudinal grating displacement from focus, $d$ is the grating pitch, $\lambda$ is the wavelength, and $n$ is an integer. When displaced from a Talbot plane, or in the presence of large wavefront aberrations, the appearance and contrast of the fringes may change, adversely affecting wavefront measurement accuracy.

Analysis of the shearing data involves reconstruction of the test wavefront from a pair of orthogonal gradient measurements. This can be challenging to perform with high precision and accuracy. The cross-grating configuration allows the separate horizontal and vertical wavefront gradients to be measured simultaneously, eliminating a potential error source that comes from one dimensional analysis. The Fourier-transform method of interferogram analysis [21] (with or without phase-shifting) is used to selectively extract derivative measurements in the two directions. Reconstruction can be performed in a number of ways [22,23]. Especially important in high-NA measurement, care must be taken to ensure that any variations in the shear magnitude caused by the changing angle of incidence across the grating are accounted for in the analysis. Furthermore, grating tilts must be uncovered through separate tests.

Because the grating pitch is a free parameter in the design, the shear magnitude, and hence the sensitivity of the interferometer can be adjusted. The wavefront is calculated only over the sub-domain of the pupil where multiplebeam overlap occurs. This excludes areas near any domain boundary, including spiders and a central obscuration if present, and the outer edges of the pupil. While a large shear value increases the sensitivity of the measurement a larger fraction of the pupil must be excluded from the analysis. Our interferometer designs typically include several different cross gratings, with different pitch values, arranged in different regions of the window, so the appropriate grating can be selected. In our experience with nearly-diffraction-limited EUV optics, ratios of the shear angle, $\sin ^{-1} \lambda / d$, to the $N A$ angle in the $1-5 \%$ range form a good compromise to the above considerations.

Besides the uncertainties introduced by noise-sensitivity in the reconstruction of the test wavefront from its measured gradients, one significant complication for high-accuracy shearing may come from noise limitations. In some cases, the a specific level of accuracy in the measurment of a given aberration, requires a much higher accuracy measurement of its derivative aberrations.

\section{CONCLUSION}

To date, EUV interferometry with sub- $\AA$ accuracy has been performed on nine different optical systems, in three 
design categories - performance and system data are shown in Table 1. Where coherent flux is available, the techniques described here may be applied to a variety of short-wavelength optical testing applications where diffraction-limited or nearly diffractionlimited imaging is possible: these include diffractive optical elements, such as Fresnel zoneplates, and advanced glancing incidence optics.

We have found that a successful approach to optical testing begins with knife-edge, or Foucault, tests to establish the focal plane position. The LSI grating can even be used for these tests if the grating pitch is much larger than the focal spot size. LSI can be used during all stages of the system alignment to provide rapid feedback with low sensitivity to small changes in the focal plane position. PS/PDI should be used only when the system is close to optimal alignment, for high accuracy verification of the shearing measurements, and evaluation of the wavefront across the full pupil area.

\section{ACKNOWLEDGMENTS}

This work would not be possible without the expert contributions of Center for X-Ray Optics members Kevin Bradley, Brian Hoef, René Delano, Ronald

the EUV Limited Liability Corporation (LLC), and by the U.S. Department of Energy.

\section{REFERENCES}

1. Goldberg, K. A., Tejnil, E., Lee, S. H., Medecki, H., Attwood, D. T., Jackson, K. H., and Bokor, J., "Characterization of an EUV Schwarzschild objective using phase-shifting point diffraction interferometry," in Emerging Lithographic Technologies, edited by D. E. Seeger, Proc. SPIE 3048, Santa Clara, CA, 1997, pp. 264-270.

2. Goldberg, K. A., Naulleau, P., Batson, P., Denham, P., Chapman, H., and Bokor, J., J. Vac. Sci. \& Technol. B 18, $2911-2915$ (2000).

3. Sweeney, D. W., Proc. SPIE 5037 (2003), unpublished.

4. Attwood, D. T., Naulleau, P., Goldberg, K. A., Tejnil, E., Chang, C., Beguiristain, R., et al., IEEE J. Quant. Elec. 35, 709-720 (1999).

5. Medecki, H., Tejnil, E., Goldberg, K. A., and Bokor, J., Opt. Lett. 21, 1526-1528 (1996).

6. Naulleau, P. P., Goldberg, K. A., and Bokor, J., J. Vac. Sci. \& Technol. B 18, 2939-2943 (2000).

7. Mercére, P., Idir, M., Zeitoun, P., Levecq, X., Dovillaire, Bucourt, S., G., et al., "X Ray wavefront Hartmann sensor," these proceedings.

8. Sugisaki, K., Zhu, Y., Gomei, Y., and Niibe M., "ASET development of at-wavelength phase-shifting point diffraction interferometer," in Emerging Lithographic Technologies VI, edited by R. L. Engelstad. Proc. SPIE 4688, Santa Clara, CA, 2002 , pp. 695-701.

9. Naulleau, P. P. and Goldberg, K. A., Appl. Opt. 38, 3523-3533 (1999).

10. Naulleau, P. P., Goldberg, K. A., Lee, S. H., Chang, C., Attwood, D., and Bokor, J., Appl. Opt. 38, 7252-7263 (1999).

11. Goldberg, K. A., Naulleau, P., Denham, P., Rekawa, S. B., Jackson, K., Anderson, E. H., et al., "EUV Interferometry of the 0.3 NA MET Optic," in Emerging Lithographic Technologies VII, edited by R. L. Engelstad, Proc. SPIE 5037, Santa Clara, CA, 2003 , pp. 69-74.

12. Goldberg, K. A., Naulleau, P., Batson, P. J., Denham, P., et al., "EUV Interferometry of a Four-Mirror Ring-Field EUV Optical System," in Emerging Lithographic Technologies IV, edited by E. A. Dobisz, Proc. SPIE 3997, Santa Clara, CA, 2000, pp. 867-873

13. Naulleau, P., Goldberg, K. A., Tejnil, E., U.S. Patent No. 6,195,169 (2001).

14. Goldberg, K., "Extreme Ultraviolet Interferometry,” Doctoral Dissertation, Physics Department, Univ. Calif. Berkeley, 1997.

15. http://www-cxro.lbl.gov/optical_constants/, August 24, 2003.

16. Anderson, E. H., Olynick, D., Harteneck, B., Veklerov, E., et al., J. Vac. Sci. \& Technol. B 18, 2970-2975 (2000).

17. TEMPEST $3 D$ was developed by the A. Neureuther group, Department of EECS, Univ. Calif. Berkeley.

18. Goldberg, K. A., Naulleau, P., Batson, P., Denham, P., Chapman, H., and Bokor, J., J. Vac. Sci. \& Technol. B 18, 2911-2915 (2000).

19. Goldberg, K. A. and Naulleau, P., U.S. Patent No. 6,118,535 (2000).

20. Goldberg, K. A., Naulleau, P., and Bokor, J., Appl. Opt. 41, 4477-4483 (2002).

21. Bone, D. J., Bachor, H.-A., and Sandeman, R. J., Appl. Opt. 25, 1653-1660 (1986).

22. Rimmer, M. P., Appl. Opt. 13, 623-629 (1974).

23. Harbers, G., Kunst, P.J., Leibbrandt, R. Appl. Opt. $356162-6172$ (1996). 